\title{
The mechanism of the emergence of Cotesia kariyai (Hymenoptera: Braconidae) larvae from the host
}

\author{
YutAKa NAKAMATSU ${ }^{1}$, ToshiHARU TANAKA ${ }^{1}$ and JeFFrey A. HARVEY ${ }^{2}$ \\ ${ }^{1}$ Applied Entomology, Graduate School of Bio-Agricultural Sciences, Nagoya University, Chikusa, Nagoya 464-8601, Japan; \\ e-mail: nakamatu@nuagr1.agr.nagoya-u.ac.jp \\ ${ }^{2}$ NIOO-KNAW, Centre for Terrestrial Ecology, Boterhoeksestraat 48, P.O. Box 40, 6666 ZG Heteren, Netherlands
}

Key words. Braconidae, Cotesia kariyai, Noctuidae, Pseudaletia separata, emergence, emergence behavior, haemolymph volume, parasitoid, anchor

\begin{abstract}
At maturity, the endoparasitoid larvae of several subfamilies of the Braconidae have to emerge from inside of the host to pupate. Although the hosts hormonal milieu and the timing of larval parasitoid emergence have been studied, no report has yet focused on the physiological state of the host in connection with the emergence behavior of endoparasitoids. We investigated the mechanism of larval emergence behavior in a gregarious endoparasitoid, Cotesia kariyai. The parasitoid larvae inserted their mandibles into the host cuticle and perforated the integument by moving their head-capsule backwards and forwards. The emerging parasitoid larva must have a physical support (an "anchor") with the terminal appendages in order to exert the necessary pressure to cut the host integument. Morphological observations revealed that each parasitoid larva was enveloped in a capsule just before emerging from their host. Eight and nine day-old parasitoid larvae secreted material around their bodies to form these capsules. This material consisted of acid-glycoproteins which coated the exuvium of the $2^{\text {nd }}$ instar larvae. The haemolymph volume of the parasitised host also decreased in later stages and was dramatically reduced immediatly prior to parasitoid emergence. This final reduction of the host haemolymph volume is the result of absorption by parasitoid larvae. This mechanism allows the parasitoid larvae to create an anchor more easily. The parasitoid larvae could also adhere to each other with the glycoprotein. In addition, these capsules prevent the leaking of host haemolymph through the emergence hole; these holes on the host integument were plugged by the capsules after parasitoid emergence. Although the pressure acquired by the anchor was lost once the head of the parasitoid larvae emerges from the host integument, the parasitoid larvae crawls out of the host cavity using backward pointing spines which enable the parasitoid to grip the capsule and move forward via peristaltic contractions.
\end{abstract}

\section{INTRODUCTION}

The larvae of some species of hymenopteran endoparasitoids (e.g. microgastrine Braconidae) emerge from the host prior to pupation (Askew, 1971). The mature parasitoid larvae usually make individual holes on the ventral surface of the host from which they crawl out (Eastham, 1929; Godfray, 1994). During this process, little or no host haemolymph leaks from the host wound after parasitoid emergence as the exuvium of $2^{\text {nd }}$ instar larvae is left on the host surface as a plug (Fulton, 1940). Manduca sexta Linnaeus (Lepidoptera: Sphingidae) larvae parasitised by Cotesia congregata Say (Hymenoptera: Braconidae) do not die immediately after the parasitoid larvae emerge but remain alive and carry the parasitoid cocoons on their dorsum until adult parasitoid eclosion (Beckage, 1997). The parasitised host would probably die within a few hours should a large quantity of host haemolymph leak out of the host with the emerging parasitoid larvae.

Larvae of the gregarious endoparasitoid Cotesia kariyai Watanabe (Hymenoptera: Braconidae) emerge on about the tenth day after parasitisation from the host Pseudaletia separata Walker (Lepidoptera: Noctuidae) when maintained under long day conditions at $25 \pm 1{ }^{\circ} \mathrm{C}$ (Tanaka et al., 1992; Nakamatsu et al., 2001). The cocoons of the parasitoid larvae are formed when they are physically separated from the host because the host moves forward while the parasitoid larvae emerge and commence cocoon spinning. This movement of the host can only occur if host haemolymph does not leak through the emergence wounds (unpubl. data). Some researchers have focused on the hormonal milieu of endoparasitoids as this is crucial to the timing of larval parasitoid emergence (Horton \& Moore, 1993; Godfray, 1994; Poulin, 1995; Adamo et al., 1997). However, no data on parasitoid emergence behaviour or on the associated change of the parasitised host haemolymph volume are currently available.

In this paper, macro- and microscopic observations of the emergence behaviour of $C$. kariyai larvae are described. The change of the parasitised host haemolymph volume is measured to determine if it is the primary factor triggering the parasitoid to emerge from the host.

\section{MATERIAL AND METHODS}

\section{Insect rearing}

The endoparasitoid C. kariyai (Watanabe), which emerged from $P$. separata (Walker) larvae collected from cornfields in Kanoya, Kagoshima Prefecture, was maintained in our laboratory. Adult wasps were kept for mating under long day conditions at $25 \pm 1{ }^{\circ} \mathrm{C}$ with a $20-30 \%$ sugar solution as food in a glass tube $(12 \mathrm{~cm}$ long $\times 5 \mathrm{~cm}$ in diameter) for $2 \mathrm{~d}$. Fifth instar larvae of $P$. separata were used for parasitisation; hosts were stung once by parasitoids to avoid superparasitism. In addition, a laboratory colony of the Pseudaletia host was established 

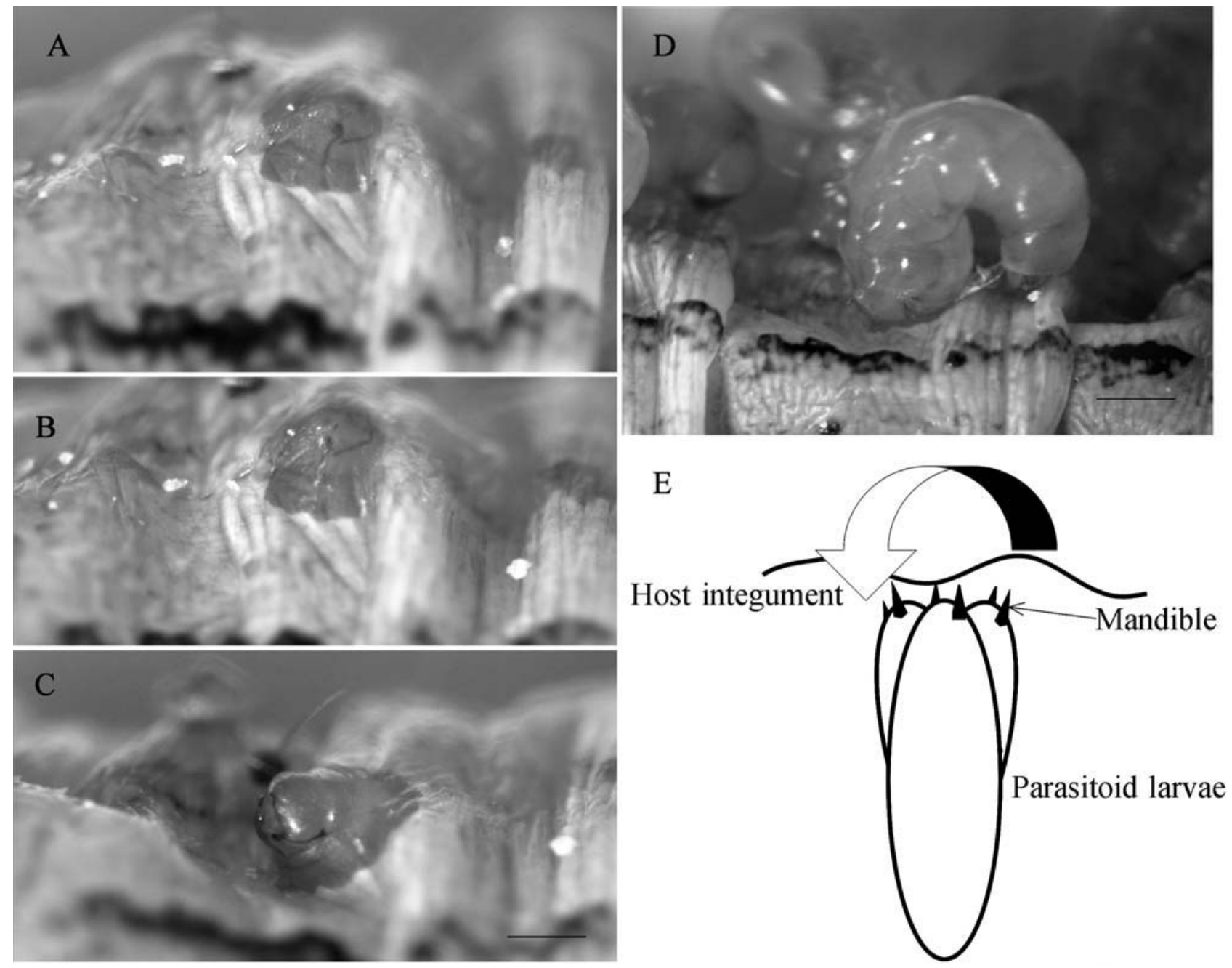

Fig. 1. A-C: C. kariyai larvae are inserting their mandibles into the host integument just prior to full emergence. The scale bar shows $500 \mu \mathrm{m}$. D: The parasitoid larvae starting to spin cocoons when the $6^{\text {th }}$ abdominal segment of the parasitoid larvae has just emerged from the host body. The scale bar shows $500 \mu \mathrm{m}$. E: Parasitoid emergence behavior. $3^{\text {rd }}$ stage parasitoid larvae just prior to emerging from the host have inserted their mandibles into the host integument and moved their heads backwards and forwards to cut the host cuticle and create a perforation hole from the inside.

using larvae collected from cornfields at the Agricultural Field Station of Nagoya University. The P. separata larvae were reared on an artificial diet (Insecta LF®, Nihon Nosan Co., Kanagawa) under a long day regime $(16 \mathrm{~L}: 8 \mathrm{D})$ at $25 \pm 1^{\circ} \mathrm{C}$. The day on which each larva ecdysed to the next instar was designated as day 0 . The $P$. separata larvae parasitised by $C$. kariyai were also maintained under the conditions described above.

\section{Observation of the parasitoid emergence behavior}

Newly molted fifth instar host larvae were parasitised and the emerging behaviour of the parasitoid larvae $10 \mathrm{~d}$ postparasitisation was observed by using a Digital Microscope (VH-60, Keyence, Osaka).

\section{Integument of 3rd stadium parasitoid larvae}

A Scanning Electron Microscope was used to observe structures on the surface of the parasitoid integument. On emergence the parasitoid larvae were fixed with $2.5 \%$ glutaraldehyde in phosphate-buffered saline (PBS; $0.87 \% \mathrm{NaCl}$ in $0.067 \mathrm{M}$ phosphate buffer, $\mathrm{pH}$ 7.4), post-fixed with $1 \% \mathrm{OsO}_{4}$ for $1 \mathrm{~h}$ and then dehydrated in an ethanol series. The samples were dried with a critical point dryer (HCP-2, Hitachi), coated with goldpalladium by Ion Sputter (E-1030, Hitachi), and observed with a Scanning Electron Microscope (S-2300, Hitachi).

\section{Observation of parasitoid larvae in the host}

Morphological observations in the body cavity of the parasitised hosts were performed to investigate the structure of the anchor. Parasitisation was performed on day 0 of $5^{\text {th }}$ instar host larvae. Host larvae $10 \mathrm{~d}$ after parasitisation (at the moment of emergence from the host) and the parasitoid larva $9 \mathrm{~d}$ after parasitisation (one day prior to emergence from the host) were fixed with $2.5 \%$ glutaraldehyde and postfixed with Bouins solution for $24 \mathrm{~h}$ then dehydrated and embedded in paraffin. Eight $\mu \mathrm{m}$ sections were stained with Mayers hematoxylin and $1 \%$ eosin Y solution (Sano, 1965).

The acid mucoglycoprotein and glycoprotein were detected with alcian blue stain and PAS stain. Eight $\mu \mathrm{m}$ sections were stained with $1 \%$ alcian blue (Merck) in 3\% acetic acid (Sano, 1965). Periodic acid Schiff reaction (PAS) stain was performed using Schiff reagent according to Sano (1965). Schiff reagent was prepared as follows: $1 \mathrm{~g}$ of basic fuchsin dye (Merck) was dissolved in $200 \mathrm{ml}$ of boiling distilled water. $20 \mathrm{ml}$ of $1 \mathrm{~N} \mathrm{HCl}$ was added when the solution was cooled down to $50^{\circ} \mathrm{C}$ and $1 \mathrm{~g}$ of Sodium persulphite was added when cooled down to $25^{\circ} \mathrm{C}$. 


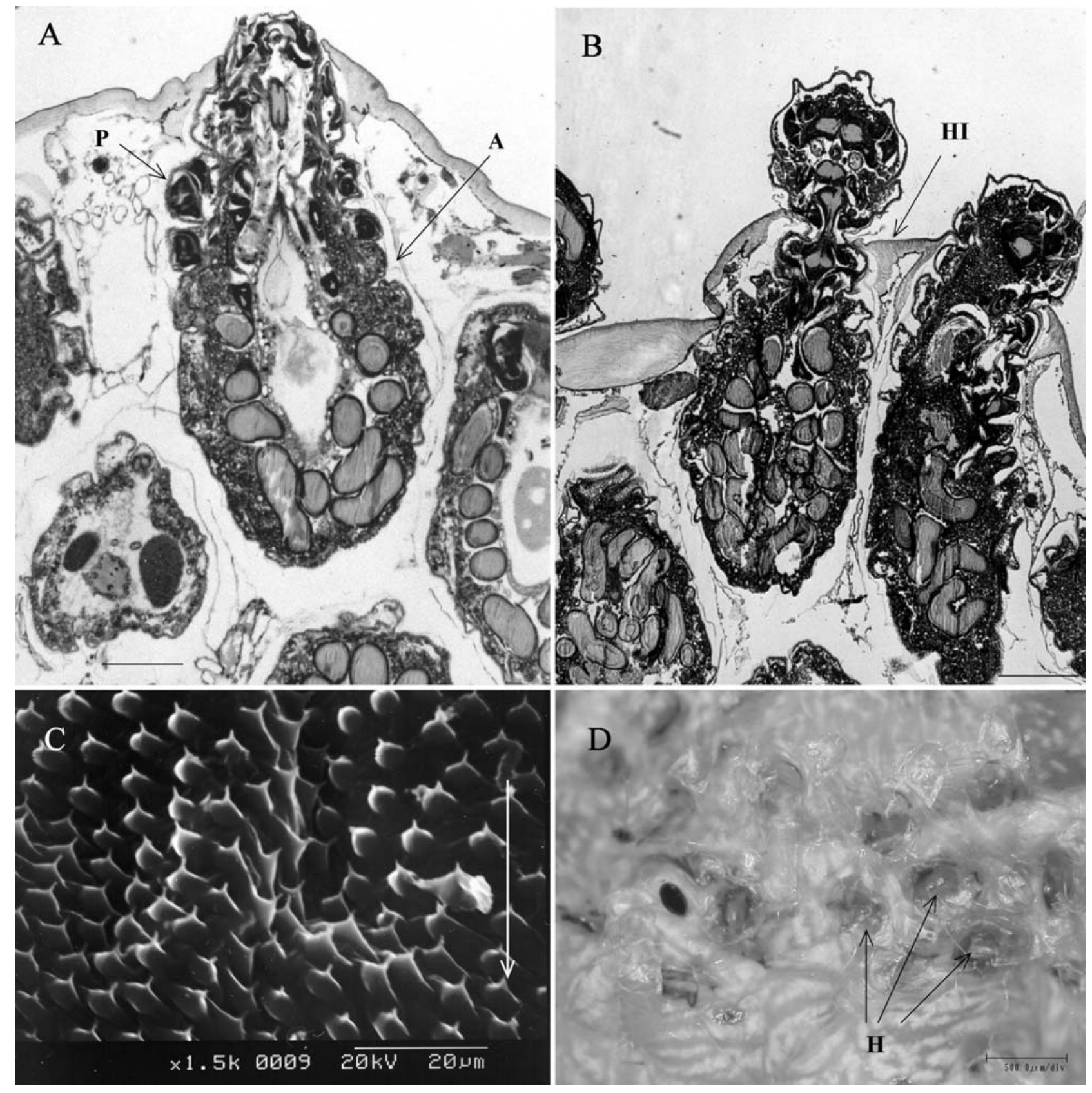

Fig. 2. A: The parasitised larva $(\mathrm{P})$ is beginning to emerge from the host. The parasitoid larva is enclosed by an anchor (A). The parasitised host was pre-fixed by injection of $2.5 \%$ glutaraldehyde solution and post-fixed with Bouins solution for $24 \mathrm{~h}$. An eight $\mu \mathrm{m}$ section was stained with Hematoxylin and Eosin dyes. The scale bar shows $500 \mu \mathrm{m}$. B: The parasitoid larvae crawl out from the host by griping on the host integument (HI) and by using spines on the cuticle of the parasitoid larva. The scale bar shows $500 \mu \mathrm{m}$. C: Many spines are found on the integument of the $3^{\text {rd }}$ stage parasitoid larva. The arrow shows the forward direction of parasitoid larvae. D: The holes $(\mathrm{H})$ were plugged by the exuvium of $2^{\text {nd }}$ instar after parasitoid emergence.

The tissues that stained to the Schiff reagent were judged as PAS-positive.

\section{Haemolymph leakage during parasitoid emergence}

The following observation was undertaken to ascertain another possible function of the anchor: Twenty day 0 fifth instar larvae were parasitised. Immediately after the emergence of parasitoid larvae, each parasitised host was placed on white filter paper (No. 2, $9 \mathrm{~cm}$ in diameter) and checked for any indication that haemolymph had exuded through the emergence holes.

\section{Weight of unparasitised and parasitised hosts}

All unparasitised or parasitised hosts were weighed individually each day at $1400 \mathrm{~h}$ using an analytical balance (Sartorius, resolution: $0.1 \mathrm{mg}$ ).

\section{Determination of haemolymph volume in the host}

The quantity of the host haemolymph was measured according to method of Weinberg (1980). Larvae were injected with four $\mu 1$ of amaranth dye solution through the second proleg after narcotizing with $\mathrm{CO}_{2}$ by a fine glass syringe. $4 \mu \mathrm{l}$ dye solution (five $\mu \mathrm{g}$ amaranth per $\mu \mathrm{l}$ ) was injected into each host larva. After 3-5 min, the haemolymph was collected from the prolegs on to cooled parafilm (American Can Co.). $980 \mu \mathrm{l}$ of $0.5 \%$ 

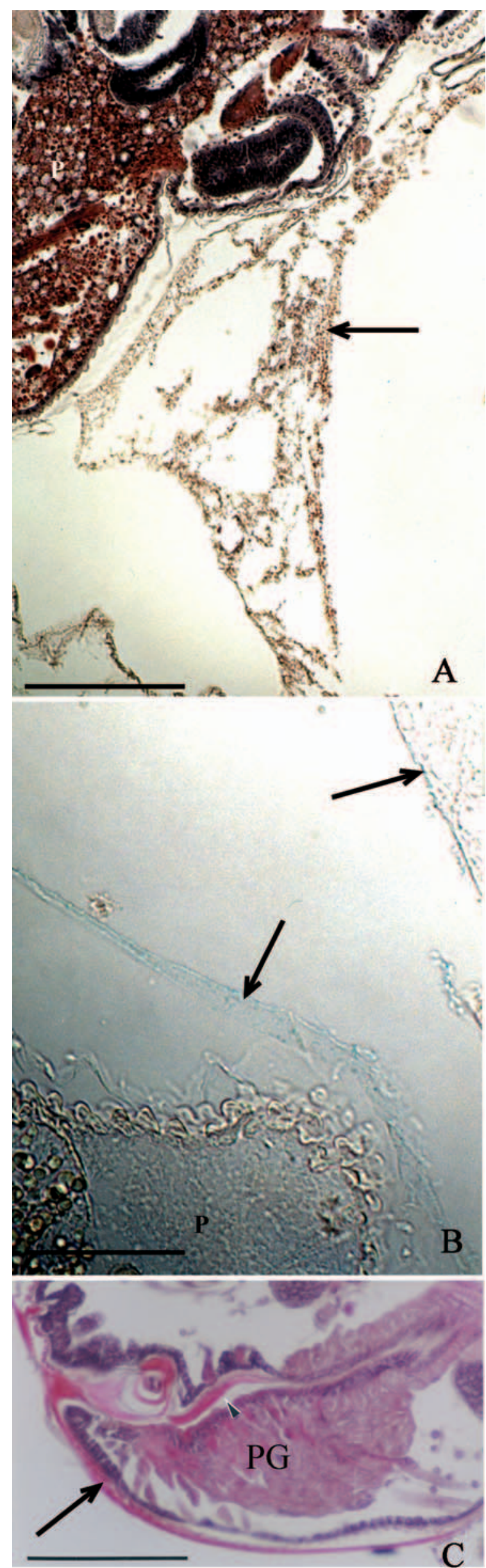

SDS-PBS solution was added after $20 \mu 1$ of dyed haemolymph was transferred to a plastic tube and the concentrations read on a spectrophotometer at $515 \mathrm{~nm}$. Standards containing from 0.5 to $40 \mu \mathrm{g}$ dye and $20 \mu 1$ haemolymph of control larvae per one $\mathrm{ml}$ of SDS solution were also measured.

Determination of whether decreasing haemolymph host volume affects the timing of parasitoid emergence from the host

Just prior to parasitoid-emergence (approx. 10 days after parasitisation), the parasitised host was bled by cutting an abdominal leg and the wound then ligated. The average haemolymph volume drawn out was $98.1 \pm 17.9 \mu$ l. An abdominal leg of sham-operated controls was ligated prior to cut. Other controls were ligated only.

\section{RESULTS}

\section{Emergence behavior of $C$. kariyai}

At maturity, third instar larvae of $C$. kariyai put their mandibles on the host integument and moved their headcapsule in a dorsal to ventral direction in order to perforate the host integument (Figs 1A-D). As a result, the host integument was linearly opened by the mandibles of the parasitoid larva, which then emerged through the resulting hole (Fig. 1D). After the headcapsules of the parasitoid larva passed through the perforation hole in the host integument the parasitoid larva crawled out of the host using spines on their body surface (Figs 2A-C). Spinning behavior (cocoon production) was initiated when the 6th abdominal segment of the parasitoid larva had emerged from the host body (Fig. 1D).

\section{Exuvium of 2nd stadium parasitoid larva}

Parasitoid larvae shed the skin of the $2^{\text {nd }}$ instar immediately prior to emergence. The $3^{\text {rd }}$ instar retained the old cuticle of the $2^{\text {nd }}$ instar before emergence from the host (Figs 2A-B). Third instar larvae have spines which are oriented backwards and emerge from the host using peristaltic movements, and discard the exuvium of $2^{\text {nd }}$ instar after passing through the emergence hole (Fig. 2D).

\section{Structure of the anchor for larval emergence}

Cotesia kariyai larvae made perforation holes through which they emerged by cutting the host integument. The parasitoid larva employed an anchor in order to orient its body for lifting up through the host integument. Before molting to $3^{\text {rd }}$ stage larvae, the pharate $2^{\text {nd }}$ stage remained adhered to each other by a sticky material. This material showed a positive reaction for the periodic acid Schiff reaction (PAS) (Fig. 3A) and was stained by alcian blue (Fig. 3B), suggesting that it is an acid-glycoprotein. The day 8 and 9 parasitoid post-parasitisation larvae secreted a substance stained with eosin Y dye (Fig. 3C).

\section{No haemolymph leakage on parasitoid emergence}

No haemolymph from any of the twenty parasitised host larvae was found to exude at the time of parasitoid emergence.

Fig. 3. A: PAS-positive substances around the parasitoid larva (P) in the host body cavity. B: Adhesive substances around the anchor (arrows) were stained with Alcian blue. C: Day 8 postparasitisation of parasitoid larvae. The substance was secreted from pharyngeal gland (PG) of parasitoid larvae (shown by arrow) and was well stained by Eosin dye. 


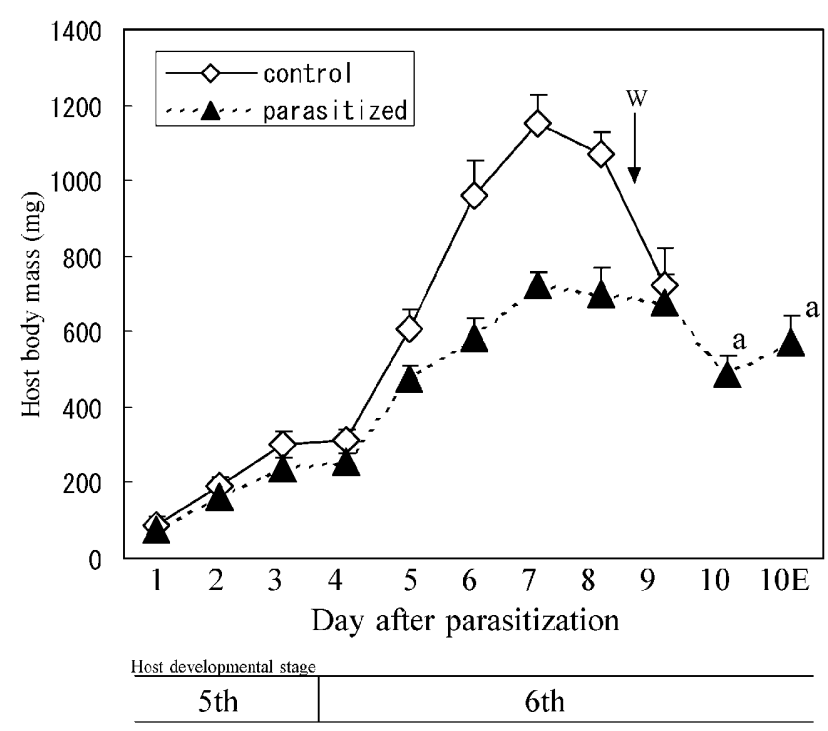

Fig. 4. Body mass of parasitised host and non-parasitised control. Parasitisation occurred on day 0 of fifth instar larvae of $P$. separata. W: Wandering stage of control larvae. 10E: Just before parasitoid emergence. Vertical bars indicate standard deviation. Different letters on the shoulder of day 10 and $10 \mathrm{E}$ point indicate significant differences (One-way ANOVA $\mathrm{p}<$ $0.01)$

Body mass and haemolymph volume of the parasitised host

The body mass of parasitised hosts was consistently lower than that of nonparasitised larvae (Fig. 4). The haemolymph volume of nonparasitised hosts increased until day 6, after which time it decreased until day 9 (Fig. $5)$. On the other hand, the haemolymph volume of the parasitised hosts increased until day 7 after parasitisation,

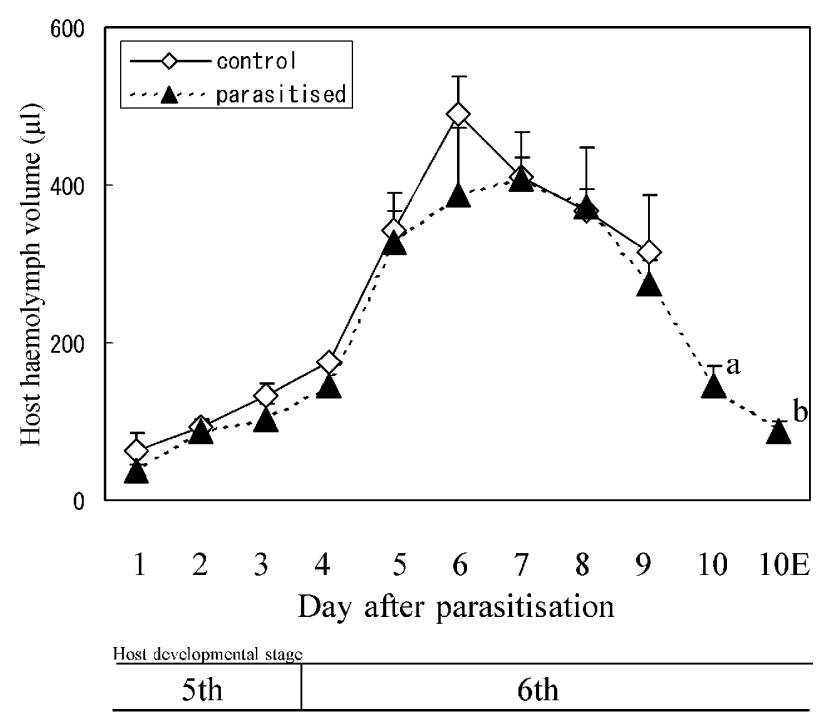

Fig. 5. Haemolymph volume of parasitised and nonparasitised host. 10E: Just before parasitoid emergence. Vertical bars indicate standard deviations. Different letters above the points for day 10 and $10 \mathrm{E}$ indicate significant differences (One-way ANOVA p < 0.01)

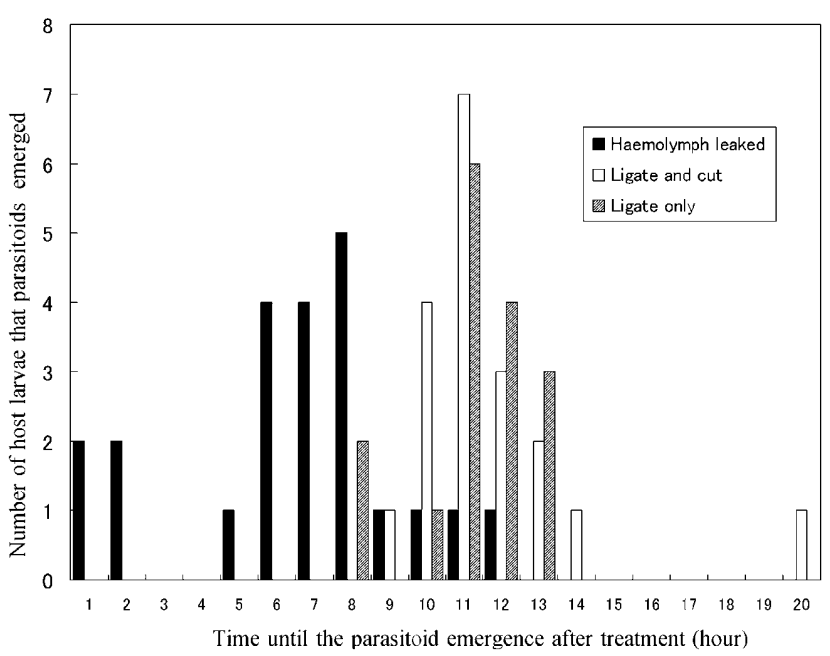

Fig. 6. The acceleration of the timing of parasitoid emergence from the host. The abdominal leg off 22 parasitised host were cut to bleed haemolymph. Ligation by cotton thread (black bars) prevented excessive haemolymph loss. Average volume of haemolymph bleed was $98.1 \pm 17.9 \mu 1$. Twenty parasitised larvae were ligated and an abdominal leg cut (white bars). No loss of haemolymph was observed. A further 16 parasitised hosts were ligated but not cut (dashed bar).

but after that time it decreased until just prior to parasitoid emergence (Fig. 5). Immediately prior to parasitoid emergence (day 10E), the mass of parasitised hosts did not decrease and was maintained at the same level as on day 10 (Fig. 4). Moreover, none of the haemolymph was observed leaking from the parasitised host. The host haemolymph volume just prior to parasitoid emergence (day 10E) clearly decreased in comparison with day 10 , showing that the parasitoid larvae actively decreased the host haemolymph volume by absorbing it into the parasitoid body.

Artificial bleeding of haemolymph from parasitised hosts just prior to parasitoid emergence accelerated the timing of the emergence (Fig. 6).

\section{DISCUSSION AND CONCLUSIONS}

In the case of many endoparasitoids, such as wasps in the higher Microgastrinae, mature larvae emerge from the host in order to pupate in a dry environment (Godfray, 1994; Harvey et al., 2000). However, no studies have thus far examined the mechanisms which enable the parasitoid larvae to make holes in the host integument, nor what strategies they use to minimize the time it takes to emerge and pupate successfully. Parasitoid larvae of C. kariyai individually made perforation holes and emerged from inside the host body cavity (Fig. 1A-D). Using an anchor enabled the parasitoid larvae to increase the physical pressure in cutting through the host integument. (Fig. 2A, B). The anchor consisted of both the old cuticle of the $2^{\text {nd }}$ stage $C$. kariyai larvae and a sticky adhesive substance secreted by the larvae (Fig. 3A, B). This substance, which is much like an acid-glycoprotein, appears to act as a glue that adheres the larvae to each other just before emergence (Fig. 3C). 
However, the pressure acquired by pressing against the anchor was lost once the head of the parasitoid larva had emerged through the host surface. The $3^{\text {rd }}$ stage parasitoid larva crawled out from the host by employing peristaltic forward movements, which were facilitated by the contraction and expansion of small bands running across the many folds in the skin of the musculature (Wigglesworth, 1972). The integument of $3^{\text {rd }}$ stage larvae contains many spines which are oriented towards the animal's posterior (Alleyne \& Beckage, 1997; Fig. 2C). The parasitoid larvae move forward using these spines which are forced into the host integument. The spines also prevent the larva from sliding back into the host.

Mature larvae of $C$. congregata also make holes in the integument of $M$. sexta before emerging from the host (Fulton, 1940; Beckage, 1997). However, despite the fact that they do not feed, the host larvae are able to remain alive for many days after the emergence of the parasitoid larvae. This is because there is no leakage of haemolymph and sufficient fat body, necessary for metabolic activity, remains inside the host larva. In the experimental $C$. kariyai - P. separata association, the newly emerged parasitoid larvae were pulled out artificially with forceps and a significant amount of haemolymph leaked from the host leading to rapid death. As a result, the parasitoid larvae were unable to make normal cocoons (Y. Nakamatsu, unpubl. data). Fulton (1940) reported that the exuvium of the $2^{\text {nd }}$ instar larvae of $C$. congregata effectively prevents the flow of blood from the host. Furthermore, in the C. kariyai - P. separata association the exuvium of $2^{\text {nd }}$ instar parasitoid larvae is clearly used to plug the perforation holes in combination with a glycoprotein. The glycoprotein is not only used to form the anchor in the host body cavity, but also plays a critical role by glueing the exuvium to the host integument (Fig. 2D). This important function prevents the host haemolymph from leaking even when the parasitoid larvae have perforated the integument.

The haemolymph volume of full-grown $5^{\text {th }}$ instar larvae of Bombix mori decreases with development (Nagata et al., 1980). Similarly, the haemolymph volume of nonparasitised $P$. separata larvae also decreased after reaching the full-grown size just prior to pupation (Fig. 4). Moreover, the haemolymph volume of parasitised $P$. separata larvae decreased to approximately half the volume of non-parasitised hosts on day 10 after parasitisation (Fig. 5). On day 10E (just prior to parasitoid emergence), the host haemolymph volume of parasitised hosts was further decreased in spite of no change in weight (Figs 4, 5). The primary factor accounting for the decrease in haemolymph volume prior to parasitoid emergence was absorption by the parasitoid larvae. The reduction of the host haemolymph volume may be apparently an adaptive mechanism that allows parasitoid larvae to construct and maintain an anchor more easily since the parasitoid larvae are brought closer together by the decreasing haemolymph volume. Beckage et al. (2002) reported that the parasitoids emergence behavior could be halted prematurely if the parasitised host was treated with $\mathrm{JH}$ or its mimic, methoprene. A release of ecdysistriggering hormone seems to be accompanied with the parasitoids haemolymph-absorption behavior. Further artificial manipulation of the haemolymph volume, which accelerated the timing of parasitoid emergence, supported the above results (Fig. 6). However, there is a possiblity that the acceleration of the emergence timing is due to the stress which is caused by the artificial reduction of the host haemolymph volume, not to the shortened distance between the parasitoid larvae.

ACKNOWLEDGEMENTS. We gratefully thank to H. Tsumuki at Research Institute for Bioresources of Okayama University for techniqual supports.

\section{REFERENCES}

Adamo S.A., Linn C.E. \& Beckage N.E. 1997: Correlation between changes in host behaviour and octopamine levels in the tobacco hornworm, Manduca sexta parasitised by the gregarious braconid parasitoid wasp Cotesia congregata. J. Exp. Biol. 200: 117-127.

Alleyne M. \& Beckage N.E. 1997: Parasitism-induced effects on host growth and metabolic efficiency in tobacco hornworm larvae parasitised by Cotesia congregata. J. Insect Physiol. 43: 407-424.

Askew R.R. 1971: Parasitic Insect. Heinemann Educational Books, London, 316 pp.

Beckage N.E. 1997: The parasitic wasps secret weapon. Sci. Am. 277: 82-87.

Beckage N.E., Foreman R.C., Palmatier C.M. \& Tan F.F. 2002: Inhibition of the larval ecdysis and emergence behavior of the parasitoid Cotesia congregata by methoprene. J. Insect Physiol. 48: 725-732.

EASTHAM L.E.S. 1929: The post-embryonic development of Phaenoserphus viator Hal. (Proctotrupoidea), a parasite of the larva of Pterostichus niger (Carabidae), with notes on the anatomy of the larva. Parasitology 21: 1-21.

Fulton B.B. 1940: The hornworm parasite Apanteles congregatus Say and the hyperparasite, Hypopteromalus tabacum (Fiych). Ann. Entomol. Soc. Am. 33: 231-244.

Godfray H.C.J. 1994: Parasitoids-Behavioral and Evolutionary Ecology. Princeton University Press, New Jersey, 473 pp.

Harvey J.A., Kadash K. \& Strand M.R. 2000: Differences in larval feeding behavior correlate with altered developmental strategies in two species of parasitic wasps: implications for the size-fitness hypothesis. Oikos 88: 621-629.

Horton D.R. \& Moore J. 1993: Behavioral effects of parasite and pathogens of insects. In Beckage N.E., Thompson S.N. \& Federici B.A. (eds): Parasites and Pathogens of Insects. Vol. 1: Parasites. Academic Press, New York, pp. 107-124.

Nagata M., Seong S. \& Yoshitake N. 1980: Variation of the haemolymph volume with larval development of the silkworm, Bombyx mori. J. Sericult. Sci. Japan 49: 453-454.

Nakamatsu Y., Gyotoku Y. \& TAnaka T. 2001: The endoparasitoid Cotesia kariyai $(\mathrm{Ck})$ regulates the growth and metabolic efficiency of Pseudaletia separata larvae by venom and $\mathrm{Ck}$ polydnavirus. J. Insect Physiol. 47: 573-584.

Poulin R. 1995: Adaptive changes in the behaviour of parasitised animals: a critical review. Int. J. Parasitol. 25: 1371-1383.

TANAKA T., Yagi S. \& NAKAmatsu Y. 1992: Regulation of parasitoid sex allocation and host growth by Cotesia (Apanteles) kariyai (Hymenoptera: Braconidae). Ann. Entomol. Soc. Am. 85: $310-316$.

SAno Y. 1965: Histological Technics. Nanzando Company, Tokyo.

WeInBERG H.L. 1980: Hemolymph volume determination in the tomato fruitworm, Heliothis zea. Experientia 36: 548-549.

Wigglesworth V.B. 1972: The Principles of Insect Physiology. Chapman and Hall, London, 827 pp.

Received July 8, 2005; revised and accepted October 25, 2005 